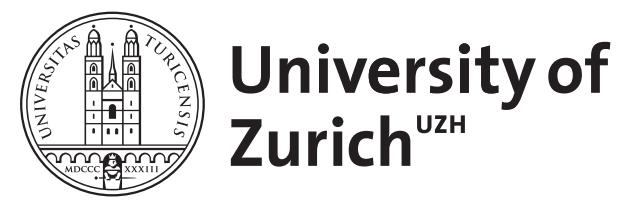

\title{
Hausaufgaben in der Paartherapie
}

\author{
Bodenmann, Guy ; Götz, Christina
}

\begin{abstract}
Hausaufgaben spielen in der Paartherapie, unabhängig von deren theoretischer Ausrichtung, eine bedeutende Rolle. Dabei variieren je nach therapeutischem Ansatz die Form, der Inhalt, die Häufigkeit und der Zeitpunkt im therapeutischen Prozess, wann Hausaufgaben aufgegeben werden. Bezüglich Funktion der Hausaufgabe gibt es deutlich weniger Differenzen und die meisten Richtungen der Paartherapie anerkennen den Nutzen von Hausaufgaben zur Gewinnung neuer Einsichten oder zur Vertiefung und Festigung von neuen Verhaltensweisen, Kognitionen oder Emotionen. Gerade in der Verhaltenstherapie mit Paaren spielen Hausaufgaben eine zentrale Rolle. Sie sind häufig inhaltlich und formal strukturiert und werden im Gegensatz zu anderen Ansätzen als verpflichtender Beitrag des Paares zum Gelingen der Therapie angesehen. Dieser Artikel versucht einen Überblick über den Einsatz und die Bedeutung von Hausaufgaben in der Paartherapie sowie über verschiedene Formen und Handhabungsmöglichkeiten zu geben. Schwierigkeiten mit Hausaufgaben im Rahmen der Paartherapie werden ebenfalls thematisiert.
\end{abstract}

DOI: https://doi.org/10.1024/1661-4747/a000105

Posted at the Zurich Open Repository and Archive, University of Zurich

ZORA URL: https://doi.org/10.5167/uzh-62493

Journal Article

Accepted Version

Originally published at:

Bodenmann, Guy; Götz, Christina (2012). Hausaufgaben in der Paartherapie. Zeitschrift für Psychiatrie, Psychologie und Psychotherapie, 60(2):103-110.

DOI: https://doi.org/10.1024/1661-4747/a000105 


\title{
Hausaufgaben in der Paartherapie
}

\author{
Guy Bodenmann \\ Christina Götz
}

Psychologisches Institut der Universität Zürich

erschienen:

Bodenmann, G. \& Götz, C. (2012). Hausaufgaben in der Paartherapie. Zeitschrift für Psychiatrie, Psychologie und Psychotherapie, 60 (2), 103-110. 
Zusammenfassung. Hausaufgaben spielen in der Paartherapie, unabhängig von deren theoretischer Ausrichtung, eine bedeutende Rolle. Dabei variieren je nach therapeutischem Ansatz die Form, der Inhalt, die Häufigkeit und der Zeitpunkt im therapeutischen Prozess, wann Hausaufgaben aufgegeben werden. Bezüglich Funktion der Hausaufgabe gibt es deutlich weniger Differenzen und die meisten Richtungen der Paartherapie anerkennen den Nutzen von Hausaufgaben zur Gewinnung neuer Einsichten oder zur Vertiefung und Festigung von neuen Verhaltensweisen, Kognitionen oder Emotionen. Gerade in der Verhaltenstherapie mit Paaren spielen Hausaufgaben eine zentrale Rolle. Sie sind häufig inhaltlich und formal strukturiert und werden im Gegensatz zu anderen Ansätzen als verpflichtender Beitrag des Paares zum Gelingen der Therapie angesehen.

Dieser Artikel versucht einen Überblick über den Einsatz und die Bedeutung von Hausaufgaben in der Paartherapie, sowie über verschiedene Formen und Handhabungsmöglichkeiten zu geben. Schwierigkeiten mit Hausaufgaben im Rahmen der Paartherapie werden ebenfalls thematisiert.

Schlagwörter: Hausaufgaben, Paartherapie, Commitment, Wirksamkeit. 


\section{Homework in couple therapy}

Abstract. Many couple therapies are aware of the importance of establishing changes occurring during the couple therapy also in daily life. Thus the important role of homework is accepted in most of currently used couple therapies, although content, form, timing and directives often vary from one approach to the other. However, all approaches agree on the fact that homework is an appropriate method to enhance and foster new insights and skills, and that the time between the therapy sessions may be used to transfer experiences made during the therapy in daily life. Homework plays the most important role in cognitivebehavioral couple therapy. In this approach, therapists require regular and committed homework of both partners in order to ensure a favorable therapy outcome. Nevertheless, also other approaches refer regularly to homework assignment.

The article tries to give an overview of homework assignment in couple therapy and to reflect function and goals of this therapeutic element. Difficulties with homework assignment in couple therapy are discussed as well.

Keywords: Homework, couple therapy, marital therapy, commitment, efficacy. 
Die Wirksamkeit von Paartherapien ist international nachgewiesen (Dunn \& Schwebel, 1995; Shadish \& Baldwin, 2005) und widerspiegelt sich in mittleren bis starken Effekten $(d=.50-1.30$, Shadish \& Baldwin, 2003; $d=.74-.95$, Shadish et al., 1993). Studien zeigen, dass rund 70\% der Paare nach einer Paartherapie eine signifikante Besserung erfahren (Baucom, Shoham, Mueser, Daiuto \& Stickle, 1998; Christensen \& Heavey, 1999), welche bei 46 bis 56\% der Paare auch noch fünf Jahre nach der Paartherapie nachweisbar ist (Christensen, Atkins, Baucom \& Yi, 2010). Während sich vor allem relativ stabile positive Veränderungen bezüglich des Verhaltens der Paare finden $(d=.52-.75$ innerhalb von zwei Jahren), sind die Effekte bei kognitiven Veränderungen schwächer $(d=.08-.18)$ und finden sich kaum mehr bei emotionalen Veränderungen (Heekerens, 2002). Offensichtlich gelingt es mittels Paartherapie besser, dysfunktionales Verhalten der Paare zu beeinflussen, als deren Kognitionen und Emotionen zu verändern. Dies dürfte damit zusammenhängen, dass alle Paartherapien, unabhängig von der Schulenorientierung, das Ziel verfolgen, die Interaktion zwischen den Partnern zu verbessern (Epstein, 2010). Dementsprechend wird in den meisten Paartherapien ein Schwerpunkt auf die Förderung der Kommunikation des Paares und damit auf eine positive Veränderung dysfunktionaler Verhaltensweisen gelegt (Bodenmann, 2004; Epstein \& Baucom, 2002; Schindler, Hahlweg \& Revenstorf, 1998). Auch wenn die Art und Weise, wie dieses Ziel erreicht werden soll, zwischen den verschiedenen Ansätzen variiert, und unterschiedliche Erklärungsmodelle für Partnerschaftsstörungen und entsprechend unterschiedliche Methoden zum Einsatz kommen, spielen doch in sämtlichen Ansätzen Psychoedukation und Übungen eine zentrale Rolle. Daher ist auch nachvollziehbar, dass in der Paartherapie, ähnlich wie in der Individualtherapie, gemeinsame Wirkfaktoren (common factors) mitunter eine größere Rolle spielen, als spezifische Interventionsmethoden (Christensen et al., 2010; Messer \& Wampold, 2002). So berichten Luborsky et al. (2002) von einer vernachlässigbaren Effektstärke von schulenspezifischen Therapieelementen $(d=.02)$ 
und Imel und Wampold (2008) resümieren, dass unspezifische Wirkfaktoren 30 bis $70 \%$ der Varianz von Paarinterventionen aufklären (siehe auch Sprenkle, Davis \& Lebow, 2009). Einer dieser unspezifischen Wirkfaktoren, welche für den Erfolg der Paartherapie mitverantwortlich sind, dürften Hausaufgaben sein. So zeigt eine neuere Metaanalyse, dass 62\% der Paare von der kognitiven Verhaltenstherapie profitierten, wenn Hausaufgaben verwendet wurden, während nur bei 38\% der Paare ohne Hausaufgaben eine Verbesserung beobachtet werden konnte (Kazantzis, Whittington \& Dattilio, 2010). Seit rund zehn Jahren wird in den meisten Paartherapien die Bedeutung von Hausaufgaben als ein wesentliches Mittel zur Konsolidierung des Gelernten zwischen den Therapiesitzungen anerkannt (Aponte \& DiCesare, 2000; Bodenmann, 2004; Dattilio \& Dickson, 2007; Dattilio, Kazantzis, Shinkfield \& Carr, 2011; Schindler et al., 1998). Während Dattilio (1998) berichtet hatte, dass 75\% der Paar- und Familientherapeuten, 16 verschiedene Ansätze repräsentierend, regelmäßig mit Hausaufgaben arbeiten, fanden Dattilio et al. (2011) rund zehn Jahre später, dass sogar 97\% der Paartherapeuten Hausaufgaben aufgeben, wovon 41\% regelmäßig und 38\% häufig. Hausaufgaben sind damit ein Instrument, das nicht nur in der verhaltenstherapeutischen Paartherapie, sondern auch in der systemischen, psychodynamischen, emotionsfokussieren, lösungsorientierten oder integrativen Paartherapie häufig zur Anwendung kommt (Aponte \& DiCesare, 2000; Dattilio et al., 2011). Je nach therapeutischem Ansatz unterscheidet sich jedoch die Art und Weise wie Hausaufgaben eingesetzt werden. Dabei variieren Inhalt, Form und Häufigkeit sowie der Zeitpunkt, bzw. wann im paartherapeutischen Prozess Hausaufgaben aufgegeben werden. Je nachdem können Hausaufgaben vom therapeutischen Konzept inhaltlich und zeitlich im Ablauf der Therapie vordefiniert sein, oder aus den Sitzungsinhalten ad hoc entstehen und von der Situationsdynamik abgeleitet werden. Dattilio et al. (2011) fanden, dass zwar die meisten Paartherapeuten mit Hausaufgaben arbeiten, dass kognitiv-verhaltenstherapeutische Paartherapeuten innerhalb der ersten zehn Sitzungen jedoch 
signifikant häufiger auf dieses therapeutische Mittel zurückgreifen als Therapeuten mit anderen Ansätzen.

Um einen Überblick über den Einsatz und die Bedeutung von Hausaufgaben in der Paartherapie geben zu können, soll zuerst geklärt werden, was darunter verstanden wird, und welche Zielsetzungen sie im allgemeinen Therapieprozess haben.

\section{Definition von Hausaufgaben in der Paartherapie}

Grob definiert handelt es sich bei Hausaufgaben um geplante therapeutische Aktivitäten, welche der Therapeut mit dem Paar vereinbart und die vom Paar zwischen den geplanten Sitzungen durchgeführt werden, um zielgerichtet Veränderungs- und Aufrechterhaltungsprozesse zwischen den Therapiesitzungen zu stimulieren (Kazantzis \& L'Abate, 2006).

Unter Hausaufgaben in der Paartherapie verstehen Epstein und Baucom (2002) Extensionen von Therapiesitzungen zur Veränderung von Verhalten, Kognitionen und Emotionen mittels zwei Arten von Aktivitäten, welche der Therapeut mit dem Paar während einer Therapiestunde plant und auf die nächste Sitzung aufgibt: (1) Hausaufgaben zur angeleiteten Verhaltensänderung und (2) Hausaufgaben zum Aufbau neuen Verhaltens. Beim ersten Typ geht es darum, funktionales Verhalten in der Beziehung, welches das Paar früher bereits praktizierte jedoch im Verlaufe der Zeit in Vergessenheit geraten ließ und nun zum Gelingen der Partnerschaft wieder häufiger zeigen sollte, in seiner Auftretenswahrscheinlichkeit durch Übung zu erhöhen. Beim zweiten Typ soll das Paar neue Verhaltensweisen erlernen, welche bisher nicht im Verhaltensrepertoire waren. Hier soll in der Therapiesitzung zuerst eine Psychoedukation und klare Instruktion erfolgen, wie diese Fertigkeiten aussehen. Anschließend wird mittels Modelllernen oder Prompting das gewünschte Zielverhalten (z. B. Kommunikation von Gefühlen und Bedürfnissen, Zusammenfassen. empathisches Zuhören) 
aufgebaut und geübt und erst nachfolgend als Hausaufgabe gegeben (Bodenmann, 2004; Epstein, 2010; Schindler et al., 1998).

Ziel von Hausaufgaben in der Paartherapie ist somit die Festigung bereits im Verhaltensrepertoire vorhandener angemessener Verhaltensweisen und/oder der Aufbau neuer funktionaler Verhaltensweisen, welche häufiger im Alltag gezeigt werden sollen. Durch Hausaufgaben soll die Therapie zur 24-Stunden-Erfahrung werden (Dattilio \& Dickson, 2007). Theoretisch geht man dabei davon aus, dass je mehr das funktionale Verhalten durch intensives Üben trainiert wird, desto grösser wird die Gewohnheitsstärke dieses Verhaltens (Hull, 1952) und damit die Wahrscheinlichkeit, dass es den Partnern gelingt, das gewünschte Verhalten anstelle des dysfunktionalen Verhaltens auch im Alltag zu zeigen. Damit liegt eine zentrale Idee von Hausaufgaben darin, wichtige Prozesse nicht auf das therapeutische Setting und darin stattfindende Impulse und Übungen zu beschränken (Fehm \& Fehm-Wolfsdorf, 2009), sondern die Zeit zwischen den einzelnen Therapiesitzungen optimal für Lernerfahrungen zu nutzen. Einerseits soll dadurch die Dauer der Therapie verkürzt, andererseits die ökologische Validität des in der Therapiesitzung gelernten Verhaltens, indem dieses im Alltag umgesetzt und geübt wird, erhöht werden (Epstein, 2010). Erst wenn ein nachhaltiger Transfer des Gelernten in den Alltag stattgefunden hat, kann die Behandlung als erfolgreich bewertet werden. In der Paartherapie spielt dieser Punkt eine ebenso zentrale Rolle wie in der Individualtherapie, da dysfunktionale Verhaltensmuster bei Paaren in der Regel in hohem Masse eingeschliffen sind und ein erheblicher Übungsaufwand erforderlich ist, um diese dysfunktionalen Verhaltensmuster zu verändern. Dabei ist es entscheidend, dass bevor an dysfunktionalen Verhaltensweisen gearbeitet werden kann, diese gemäss dem Modell von Maccoby und Solomon (1981) bewusst gemacht und Wissen und Motivation geschaffen werden. Auch hierzu eignen sich Hausaufgaben bestens. 


\section{Formen von Hausaufgaben in der Paartherapie}

In der Paartherapie werden verschiedene Formen von Hausaufgaben verwendet, die Dattilio und Dickson (2007) folgendermaßen klassifizieren: Eine wichtige erste Kategorie bilden Selbstbeobachtungsübungen, bei denen beide Partner aufgefordert werden, sich bis zur nächsten Therapiesitzung in Form von Selbstreflexion und schriftlicher Analyse mit bestimmten Themen, Kognitionen oder Emotionen auseinanderzusetzen. Auf individueller Ebene könnten beispielsweise Aufgaben sein, sich Szenarien zu überlegen, wie die Beziehung in zehn Jahren aussehen soll und was man selber zu diesen Szenarien beitragen kann, oder die Ergründung von kognitiven Standards, Attributionsmustern, Erwartungen und Zielen. In dem Zusammenhang werden häufig Protokolle, Tagebücher oder Materialien zur kognitiven Umstrukturierung (Spaltenprotokolle) verwendet (Baucom, Epstein, LaTaillade \& Kirby, 2008; Epstein \& Baucom, 2002) oder zuhause „Experimente“ durchgeführt, bei denen die Partner ihre Erwartungen überprüfen sollen (Epstein, 2010). Ein solches Experiment könnte darin bestehen, dass die Partner aufgefordert werden, beim Zuspätkommen des Partners in der nächsten Situation nicht gleich mit Ärger zu reagieren, sondern nachzufragen, was passiert ist und warum der Partner müde oder gereizt nach Hause kommt. Diese Erfahrungen können helfen, kognitive und emotionale Prozesse zu verändern. Übungen zum Erkennen von emotionalem Erleben in den dyadischen Interaktionen im Alltag bilden insbesondere in der emotionsfokussierten Paartherapie einen häufig verwendeten Typ Hausaufgaben, wo mittels eines Emotionstagebuchs protokolliert werden soll, welches Verhalten des Partners welche Gefühle (Freude, Schmerz, Trauer etc.) bei einem selber auslöst (Greenberg \& Goldman, 2010). Weiter kann beispielsweise im Rahmen der Verhaltensbeobachtung auf dyadischer Ebene als Hausaufgabe aufgegeben werden, sich damit auseinander zu setzen, in welchen Situationen man in welcher Art und Weise mit dem Partner interagiert, welche Effekte dies hat und was man selber dazu beiträgt, dass es zum wechselseitig dysfunktionalen Verhalten kommt. Solche Beobachtungsaufgaben sind zu 
Beginn einer Paartherapie von zentraler Bedeutung, bleiben aber während der gesamten Therapie sinnvoll. Sie sollen die Partner dafür sensibilisieren, dysfunktionale Verhaltensweisen (z. B. Kommunikationsfehler sensu, Gottman, 1994) zu erkennen und sich ihrer Intention und Wirkung bewusst zu werden. Dies kann mittels Tagebuch oder Verhaltensprotokoll erfolgen, oder (falls das Paar dazu bereit ist) sogar mittels einer Audiooder Videoaufzeichnung, damit im natürlichen Kontext stattfindende Interaktionsmuster in der Therapie gemeinsam mit dem Therapeuten analysiert und besprochen werden können (Dattilio, 2002).

Die zweite Kategorie Hausaufgaben betrifft die Aktivitätenplanung. Hier wird das Paar aufgefordert, gemeinsame hedonistische Aktivitäten (z. B. gemeinsame Spaziergänge, gemeinsamer Restaurantbesuch, gemeinsame sportliche Aktivitäten.) in ihren Alltag einzuplanen und deren Verstärkerwert zu beurteilen. Diese Hausaufgabe wird gelegentlich auch durch die klassische Form des Reziprozitätstrainings ersetzt oder kann parallel dazu aufgegeben werden (Bodenmann, 2004; Schindler et al., 1998). L'Abate (2007) hat mit der Technik 3HC (3H: hugging, holding, huddling und C: cuddling) zudem einen interessanten Ansatz vorgeschlagen, um positive Aktivitäten im Alltag der Paare zu fördern. Er hält die Partner dazu an, zärtliches Verhalten vermehrt zu zeigen (mindestens ein- bis zweimal täglich je 10-20 Minuten), wobei Häufigkeit und Art des Austauschs von Zärtlichkeiten von beiden Partnern erwünscht sein und freiwillig erfolgen müssen.

Drittens kommen Verhaltensübungen zum Einsatz. So wird beispielsweise im Rahmen des Kommunikationstrainings Paaren die Hausaufgabe gegeben, die Sprecher- und Zuhörerregeln zu üben. Ein weiteres Beispiel ist die 3-Phasen-Methode zur Verbesserung des dyadischen Copings (Bodenmann, 2004), in der die Partner aufgefordert werden, zwischen den Therapiesitzungen zu trainieren sich zu erzählen, was sie tagsüber für Belastungen erfahren haben, was die Situationen bei ihnen ausgelöst haben und was genau diese so schlimm machten. Der zuhörende Partner soll dabei empathisch mitschwingen und daraufhin 
angemessene Unterstützung geben lernen. In der Verhaltenstherapie mit Paaren stellen Verhaltensübungen als Hausaufgabe eine zentrale Form dar, welche in jedem Fall zum Einsatz kommt. Auch diese Aufgabe kann zu Hause audio- oder videographiert und anschließend in der nächsten Therapiesitzung besprochen werden.

Ein viertes Element von Hausaufgaben stellen Bibliointerventionen dar. Den Paaren kann auf die nächste Therapiesitzung eine Informationsbroschüre oder die Lektüre eines Kapitels in einem Selbsthilfebuch aufgegeben werden. Im deutschen Sprachraum haben Schindler, Hahlweg und Revenstorf (1999) mit dem Selbsthilfebuch „Partnerschaftsprobleme: Möglichkeiten zur Bewältigung. Ein Handbuch für Paare“ oder Bodenmann (2005, 2006) mit seinen Büchern „Beziehungskrisen: Erkennen, verstehen, bewältigen“ respektive „Stress und Partnerschaft: Gemeinsam den Alltag bewältigen “ und Bodenmann und Fux Brändli (2011) mit „Was Paare stark macht“ solche wissenschaftlich fundierten Ratgeber publiziert, welche sich als Hausaufgabe im Sinne einer Bibliointervention eignen. Auch DVDs können hierbei eine wichtige und nützliche Rolle spielen. Ein Beispiel für eine auf DVD basierende Bibliointervention ist „Ein Kick mehr Partnerschaft“, die auf dem EPL (Ein Partnerschaftliches Lernprogramm) basiert (Thurmaier, Engl, Eckert \& Hahlweg, 1992). Ein weiteres Beispiel ist die DVD „, Glücklich zu zweit trotz Alltagsstress“ (Bodenmann, Schär \& Gmelch, 2008), der das Stresspräventionsprogramm paarlife (Bodenmann \& Shantinath, 2004) zugrundeliegt.

Schließlich kommen im Rahmen der kognitiven Paartherapie auch klassische kognitive Methoden als Hausaufgaben zum Einsatz (kognitive Umstrukturierung, Reattributionstraining, usw.), wie sie in der Individualtherapie vorgeschlagen werden.

Gemäß Dattilio et al. (2011) werden über alle Schulen hinweg am häufigsten In Vivo Expositionen (73\% oft/sehr oft), gefolgt von kognitiven Techniken (66\% oft/sehr oft) und Selbstbeobachtungsaufgaben (63\% oft/sehr oft) als Hausaufgaben aufgegeben. Bibliointerventionen (53\% oft/sehr oft) und Aktivitätenplanung (50\% oft/sehr oft) folgen auf 
den weiteren Plätzen. Erwartungsgemäß setzen kognitiv-verhaltenstherapeutische Paartherapeuten mehr kognitive Methoden, Bibliointerventionen und Verhaltenstrainings ein, dennoch erstaunt, dass auch bei den anderen Ansätzen (systemisch, psychodynamisch, integrativ usw.) die meisten Elemente ebenfalls relativ häufig zum Einsatz kommen.

\section{Funktionen von Hausaufgaben in der Paartherapie}

In der Literatur werden verschiedene Funktionen von Hausaufgaben referiert: (1) Transfer der in der Therapie behandelten Inhalte und gelernten Kompetenzen in den Alltag des Paares, wodurch ein Großteil der Therapie im ökologischen Kontext stattfindet, in dem sich das Paar bewegt und wodurch eine Generalisierung des funktionalen Verhaltens wahrscheinlicher wird. (2) Erkennen von Zusammenhängen und Aspekten in der alltäglichen Beziehungsdynamik, welche in der Paartherapie aufgedeckt und besprochen wurden und nun in der Interaktion mit dem Partner erfahren werden, wodurch in der nächsten Therapiesitzung die Möglichkeit zur weiteren vertieften Bearbeitung ermöglicht wird. (3) Die Umsetzung von in der Therapie gemachten Erfahrungen im Alltag mittels Hausaufgaben schafft eine neue Realität mit Implikationen für die individuelle und dyadische Selbstwirksamkeit. Das Paar erfährt, dass es in der Lage ist, Probleme wirksamer anzugehen, sich im Alltag konstruktiver zu begegnen und das in der Therapie Gelernte im Beziehungsalltag gewinnbringend anzuwenden. (4) Hausaufgaben erlauben ein nochmaliges Durchleben von wichtigen Therapieerfahrungen (rehearsal) mit neuen kognitiven und emotionalen Erfahrungen, was zu einer Festigung des Gelernten führen, und mittels zusätzlicher Beobachtungsübungen zu einer vertieften Diagnostik und Selbstreflexion beitragen kann. (5) Sie können auch die Motivation des Paares stärken, da sie fokussiert an einem Thema dranbleiben und dieses sowohl in den Therapiesitzungen wie auch zuhause bearbeiten und vertiefen, wodurch Kohärenz und Systematik entstehen. (6) Weiter stärkt es die Compliance des Paares, wenn beide Partner realisieren, dass der jeweils andere sich in die Hausaufgaben involviert, diese ernst nimmt und 
sich damit nachweislich für die Partnerschaft und deren Zukunft einsetzt. (7) Hausaufgaben übergeben dem Paar respektive beiden Partnern die Verantwortung bezüglich der gemeinsam definierten Therapiezielen und für die Verbesserung ihrer Beziehung, wodurch der Therapeut von Anfang an die Arbeit an der Beziehung an das Paar delegiert und seine Kräfte zur Selbsthilfe aktualisiert (Dattilio, 2002; Dattilio \& Dickson, 2007).

Allgemein steht somit nicht nur die Festigung und Generalisierung von in der Therapiesitzung modifizierten Reaktionen im Vordergrund, sondern es findet eine breitere Bearbeitung und Vernetzung von relevanten Themen und Kompetenzen zwischen Therapiesitzungen und Alltag des Paares statt. Die Zeiten zwischen den Therapiesitzungen werden durch Hausaufgaben überbrückt und verbunden und schaffen einen holistischen Ansatz, wodurch der Lerneffekt beschleunigt und effizienter gestaltet werden kann (Wuschel \& Linden, 2008). Generell können wir festhalten, dass die Hausaufgaben meist eine nachbereitende (gelegentlich auch im Vorfeld einer Sitzung für gewisse Themen sensibilisierende) Funktion haben.

\section{Handhabung von Hausaufgaben}

Während in der verhaltenstherapeutischen Paartherapie Hausaufgaben meist bezüglich des Zeitpunkts im Therapieprozess und bezüglich der Art der Hausaufgabe standardisiert sind (Bodenmann, 2004; Dattilio \& Beck, 2009; Schindler et al., 1998), werden diese in anderen Ansätzen häufig freier und unsystematischer gehandhabt (Kazantzis, Lampropoulos \& Deane, 2005). In der systemisch-lösungsorientierten Paartherapie beispielsweise, stellen Hausaufgaben (häufig als Vorschläge oder Anregungen bezeichnet), ebenfalls ein oft verwendetes Mittel dar. Sie sind aber meist nicht vordefiniert, sondern werden während der Sitzung mit dem Paar ad hoc erarbeitet und können somit von Paar zu Paar und inhaltlich variieren. Die Partner werden als Experten für ihr eigenes Anliegen und die Inhalte der Therapie verstanden, der Therapeut als Experte für die Rahmenbedingungen, weshalb 
Hausaufgaben gemeinsam mit dem Paar formuliert und nicht vom Therapeuten aufgegeben werden. Auch sehen systemisch-lösungsorientierte Paartherapeuten meist davon ab, das Paar beim nächsten Treffen auf die Erledigung der Hausaufgaben anzusprechen und fragen vielmehr in einem generellen Sinne nach, was sich seit der letzten Sitzung verändert habe. Bezug auf die Hausaufgabe wird insofern genommen, als der Therapeut nachfragt, ob die Ideen, welche in der letzten Sitzung aufgekommen sind, vom Paar auch nachträglich als nützlich und praktikabel wahrgenommen wurden (vgl. Hargens, 2010). In der emotionsfokussierten Paartherapie (Johnson, 2004) geht es vor allem um das Ausprobieren von Erfahrungen oder Verhaltensweisen, wobei die Hausaufgaben in der Regel nicht überprüft werden, sondern der Therapeut indirekt sondiert, was das Paar mit dieser Aufgabe für Erfahrungen gemacht hat und was sich dadurch in seiner Beziehung verändert hat (Greenberg \& Warwar, 2006). In der psychodynamischen Paartherapie sind Hausaufgaben meist kein fest vorgesehener Bestandteil. Sie werden aus Inhalten, die sich in der Therapie entwickeln mehr oder weniger spontan abgeleitet und dann eingesetzt, wenn der Therapeut der Meinung ist, dass sie dem Paar in der aktuellen Phase der Therapie nützlich sein könnten. Wie auch die systemisch-lösungsorientierten Therapeuten sind psychodynamische Paartherapeuten der Meinung, dass die Inhalte der Hausaufgaben gemeinsam mit dem Paar definiert werden sollten, einerseits um die Verantwortungsübernahme der Beteiligten zu steigern, andererseits aber auch, da der Therapeut sich mehr als ,aufmerksamen Begleiter“ versteht und weniger als „Anführer“ (Bauriedl, Cierpka, Neraal \& Reich, 2002). Kaum Verwendung von Hausaufgaben findet man in der klientenzentrierten Paartherapie, in welcher man davon ausgeht, dass die therapeutisch wichtigsten Momente diejenigen in der Sitzung sind. Hausaufgaben kommen in diesem Ansatz einzig dann zum Einsatz, wenn eine Therapiesitzung nicht regulär abgeschlossen werden konnte und die Weiterführung des Themas daher zuhause erfolgen soll. Die Aufgabe besteht dann darin, dass das Paar sich bis 
zur nächsten Sitzung Gedanken machen soll, was in der aktuellen Sitzung von Bedeutung war, und was als Nächstes behandelt werden sollte (Weber, 1994).

In der Verhaltenstherapie mit Paaren kommt den Hausaufgaben als systematischem und verbindlichem Element die grösste Bedeutung zu (Bodenmann, 2004; Dattilio, 2002; Schindler et al., 1998). Hier werden praktisch ab der ersten Sitzung regelmässig (meist standardisierte) Hausaufgaben aufgegeben, um die jeweils in der Sitzung behandelten Inhalte und Übungen im Alltag zu festigen. So erfordert das Reziprozitätstraining das aktive Engagement beider Partner im Alltag, indem sich in einer ersten Hausaufgabe beide Partner überlegen sollen, was sie tun können, damit sie beim anderen positives Erleben stimulieren können. In einer anschliessenden Hausaufgabe sollen die Partner sich dann bewusst werden, was der andere von diesen Aktivitäten im Alltag tatsächlich umsetzt.

Ein weiterer in der Literatur diskutierter Aspekt bei Hausaufgaben ist die Frage, zu welchem Zeitpunkt im paartherapeutischen Prozess diese aufgegeben werden sollen. Während in der Individualtherapie dazu geraten wird, dass der Schwierigkeitsgrad erst im Verlauf der Therapie gesteigert werden soll (Helbig \& Fehm, 2005), ist dies in der Paartherapie nicht so einfach. Häufig sind auch einfach wirkende Aufgaben für ein hoch dysfunktionales Paar bereit sehr schwierig umzusetzen, sodass diese Regel im Paartherapiekontext so vermutlich nicht gilt. Es ist schwierig zu beurteilen, ob Hausaufgaben im Rahmen des Reziprozitätstrainings (Aufbau von Positivität im Alltag) einfacher umzusetzen sind, als die Übung von Kommunikationsregeln. Wichtiger scheint der konkrete, direkte Bezug der Hausaufgabe zum aktuellen Therapieinhalt und die Kompatibilität der Hausaufgaben mit dem Erklärungsmodell des Therapeuten, weshalb diese dem Paar nützlich sein sollen (Bodenmann, 2004). Wie Tompkins (2002) zeigte, ist die Compliance generell höher, wenn nachvollziehbar ist, weshalb die Hausaufgaben gemacht werden sollen, man darin einen Nutzen erkennen kann und dieser mit den Therapiezielen kompatibel ist. Erst wenn beide Partner den Nutzen einer Hausaufgabe für sich selber und ihre Beziehung erkennen können, steigt die 
Wahrscheinlichkeit, dass die Hausaufgabe auch konform erledigt wird. Wichtig ist zudem, dass der Therapeut die Hausaufgaben zu Beginn der neuen Sitzung bespricht (Bryant, Simons \& Thase, 1999) und damit den Aufwand und das Engagement des Paares wertschätzt und verstärkt.

\section{Schwierigkeiten bei Hausaufgaben in der Paartherapie}

Insgesamt kann festgehalten werden, dass die meisten Paare ihre Hausaufgaben befriedigend erledigen. Dattilio et al. (2011) fanden in ihrer Studie, dass nur 7\% der an der Untersuchung teilnehmenden Paartherapeuten $(N=226)$ die Compliance der Paare als niedrig einstuften, gegenüber $61 \%$, welche eine mittlere Compliance wahrnahmen und $26 \%$, welche von einer hohen Compliance der Paare berichteten. Bezüglich der Zufriedenheit der Therapeuten mit der Qualität der erledigten Hausaufgaben zeigt sich ein ähnliches Bild: 4\% der Paartherapeuten berichteten eine niedrige Zufriedenheit, 52\% ein mittlere, 35\% eine hohe und 3\% eine sehr hohe Zufriedenheit mit der Art und Weise, wie die Paare ihre Hausaufgaben erledigt hatten.

In der Paartherapie ist es dabei besonders wichtig, dass beide Partner die Hausaufgaben erledigen und eine vergleichbare Compliance zeigen, damit Fairness zwischen den Partnern hergestellt ist. Tun sie dies nicht, muss die nicht erledigte Hausaufgabe beim säumigen Partner thematisiert und der Nutzen der Aufgabe, die Bedeutung von Fairness in der Partnerschaft als auch in der Therapie (Neutralität und Unparteilichkeit des Therapeuten; Bodenmann \& Gabriel, 2008) und mögliche Gründe und Hindernisse besprochen werden. Gemäss Dattilio et al. (2011) ist in 37\% (oft/sehr oft) der Fälle die Tatsache, dass der Partner die Hausaufgabe nicht unterstützt hat, eine Barriere zur Erledigung der Hausaufgaben. Falls beide Partner die Hausaufgaben nicht erledigen, sollte der Therapeut die Sinnhaftigkeit, das Timing, oder seine Art, die Aufgaben zu erklären hinterfragen oder aber die generelle Veränderungsmotivation des Paares nochmals grundlegend prüfen (Dattilio, 2002; Greenberg 
\& Warwar, 2006). Tompkins (2002) führt allerdings aus, dass mindestens drei Gründe für Non-Compliance bei der Ausführung von Hausaufgabenberücksichtigt werden müssen, um dem Phänomen gerecht zu werden. Dies sind aufgabenbezogene Gründe, Gründe seitens der Partner, sowie therapeutenbezogene Gründe. So darf nicht vorschnell auf Widerstand des Paares oder eines der beiden Partner geschlossen werden, was auch Jacobson und Christensen (1996) hervorheben, da oftmals inadäquate Planung, zu geringe Transparenz bezüglich des Nutzens der Aufgaben oder Überforderung aufgrund mangelnder Klarheit oder Komplexität der Hausaufgaben dahinter stehen können (Epstein, 2010). Dennoch haben die Partner durchaus häufig ihre impliziten oder expliziten Gründe, weshalb sie die Hausaufgaben nicht erledigen. Die Begründung, dass man keine Zeit fand, ist häufig eine fadenscheinige Ausrede, die so nicht gelten gelassen werden sollte. Therapierelevante im Paar liegende Ursachen sind oftmals mangelnde wirkliche Motivation die Beziehung aufrecht zu erhalten, Widerstand sich für den Partner und die Beziehung einsetzen zu müssen, von der man verletzt und enttäuscht wurde, Angst vor Veränderungen, Missmut, sich mehr engagieren zu müssen, als der Partner dies tut, die Forderung, dass der Partner den ersten Schritt tun müsse, bevor man sich auch engagiere, usw. Nicht selten entzünden sich Machtkämpfe bei der Hausaufgabenbearbeitung (Dattilio \& Dickson, 2007). Diese Gründe gilt es behutsam in der Therapie zu explorieren und mit dem Paar zu bearbeiten, damit die Hausaufgaben künftig verlässlich ausgeführt werden. Den Vorschlag, dass man anstelle des nüchternen und häufig schulisch besetzten Begriffs „Hausaufgaben“ Begriffe wie „Vorschläge“, „Tipps“ oder „Experiment““ verwenden soll, um eine höhere Motivation zu erzielen (Bevilacqua \& Dattilio, 2001; De Jong \& Berg, 2008), teilen wir nicht, da in den seltensten Fällen die Begrifflichkeit das Problem ist, sondern entweder tieferliegende Motive, welche zu einer mangelnden Compliance führen oder eine zu hohe Aufgabenschwierigkeit. Dattilio et al. (2011) berichten denn auch, dass in 90\% der Fälle die Aufgabenschwierigkeit der Grund für die Non-Compliance sei, in $87 \%$ eine negative Einstellung, in 86\% die Menge der Hausaufgaben, in 85\% der Fälle die mangelnde Klarheit 
der Hausaufgaben und in $81 \%$ der Fälle die Inkompatibilität der Hausaufgaben mit dem Weltbild des Paares (z. B. bei sexuellen Themen oder bei Paaren mit anderem kulturellen Hintergrund). Die Studie gibt allerdings keine Auskunft über Widerstände als Ursache. Wenn sich beide Partner bereit erklärt haben, die Hausaufgaben zu erledigen und diese vom Therapeuten vom Timing her passend und gut eingeführt, begründet und nachvollziehbar erklärt wurde, ist deren versäumte Ausführung häufig grundlegender Natur und diagnostisch relevant. Die Bereitschaft beider Partner zur Erledigung von Hausaufgaben kann als Gradmesser dafür gewertet werden, ob sie bereit sind, ihren Teil an der Beziehungsarbeit zu übernehmen und in die Therapie und Zukunft der Partnerschaft zu investieren, sofern ausgeschlossen werden kann, dass mangelndes Verständnis der Hausaufgabe oder Überforderung zu deren Nicht-Ausführung geführt haben. Wenn beide Partner die Hausaufgaben unzuverlässig bearbeiten oder gar sabotieren, kann eine ungünstige Prognose bereits sehr früh im Therapieprozess angenommen werden (Kazantzis et al., 2010).

\section{Indikation von Hausaufgaben in der Paartherapie}

In der Paartherapie sind keine Kontraindikationen bekannt. Allerdings ist Voraussetzung, dass beide Partner sich für die Beziehung einsetzen möchten, ein längerfristiges Commitment für die Beziehung zeigen und bereit sind, für die Partnerschaft Zeit und Energie zu investieren (Bodenmann, 2004). Bei Paaren, bei denen neben der Partnerschaftsstörung auch individuelle psychische Störungen vorliegen (affektive Störung, Suchterkrankung, Persönlichkeitsstörung usw.) ist bei der Vergabe von Hausaufgaben darauf zu achten ist, welche Hausaufgaben sinnvoll sind und wie sie allenfalls an die spezifische Konstellation angepasst werden sollten. 


\section{Wirksamkeit von Hausaufgaben}

Empirisch konnte in Individualtherapien insgesamt ein positiver Zusammenhang zwischen der Hausaufgabenvergabe und dem Therapieergebnis nachgewiesen werden. So haben Patienten, welche Hausaufgaben nicht wie vereinbart erledigen, auf der Symptomebene einen geringeren Behandlungserfolg (Helbig \& Fehm, 2005). Auch kommt es bei Patienten mit fehlender Aufgabenadhärenz zu häufigeren Therapieabbrïchen (Burns \& Auerbach, 1992). In einer Metaanalyse ergab die Berechnung für die Verwendung von Hausaufgaben über insgesamt 11 Therapiestudien hinweg eine mittlere Effektstärke von $r=.36(N=375$ Patienten; Kazantzis, 2000). Dementsprechend werden Hausaufgaben insgesamt als positiv bewertet und als unspezifischer Wirkfaktor verhaltenstherapeutischer Behandlungen diskutiert (Helbig \& Fehm, 2005). Für die Paartherapie liegen ebenfalls positive Ergebnisse vor, welche belegen, dass Paare in der Therapie mit Verwendung von Hausaufgaben besser profitieren als ohne Hausaufgaben (Kazantzis et al., 2010), allerdings ist gerade bei kognitivverhaltenstherapeutischer Paartherapie die spezifische Untersuchung der Wirksamkeit von Hausaufgaben schwierig, da diese standardmäßig zum Therapiekonzept gehören und integraler Bestandteil der Therapie sind. Epstein (2010) hält entsprechend fest, dass aufgrund dieser Tatsache Studien zur Wirksamkeit von Hausaufgaben in der Paartherapie (und dies gilt besonders für die VT) fehlen.

\section{Schlussfolgerungen für die klinische Praxis}

Zusammenfassend kann festgehalten werden, dass bei den meisten Paartherapien die Zeit zwischen den Therapiesitzungen in aller Regel dazu genutzt wird, die Inhalte der Therapiesitzungen im Alltag zu reflektieren und neue Verhaltensweisen auszuprobieren respektive durch Übung zu festigen. Das Ziel, die Zeit zwischen den Sitzungen für nachhaltige Veränderungen zu nutzen und die während der Therapiesitzung erarbeiteten Inhalte oder trainierten Kompetenzen im Alltag durch möglichst häufiges Üben zu festigen, 
ist dabei in der Paartherapie vergleichbar zur Individualtherapie. Wichtig bei Hausaufgaben in der Paartherapie ist die konsequente Ausführung durch beide Partner. Der Therapeut muss hier im Sinne der Unparteilichkeit darauf achten, dass sich beide vergleichbar investieren und die aufgetragenen Hausaufgaben ernst nehmen. Obwohl Einsatz und Form der Hausaufgaben in der Paartherapie je nach therapeutischer Richtung variieren können, erachten Paartherapeuten im Allgemeinen Hausaufgaben als nützlich und wichtig. Eine zentrale Bedeutung erhalten Hausaufgaben in der Verhaltenstherapie mit Paaren, wo sie per definitionem zum Therapiegeschehen dazugehören und standardmässig eingesetzt werden (Bodenmann, 2004; Schindler et al., 1998). Da gerade dysfunktionale Einstellungen oder Verhaltensweisen häufig eine hohe Gewohnheitsstärke (Hull, 1952) aufweisen und damit einzelne Inputs und Übungen in der Therapiesitzung an der Macht dieser „habits“ wieder scheitern und verpuffen können, zielen Hausaufgaben primär darauf ab, die Gewohnheitsstärke funktionaler Verhaltensweisen durch möglichst häufiges Üben zu erhöhen. Erst wenn das neue, funktionale Verhalten eine ähnlich hohe Gewohnheitsstärke aufweist, wie das bisherige maladaptive Verhalten, gelingt es, nachhaltige Verbesserungen zu erzielen, eingeschliffene Verhaltensmuster zu durchbrechen und neuen Verhaltensweisen im Alltag Platz zu machen. Vor diesem Hintergrund misst die Paartherapie Hausaufgaben eine ähnlich wichtige Bedeutung bei wie den Inputs in den Therapiesitzungen. Diese sollen gemäss dem Modell der Verhaltensänderung von Maccoby und Solomon (1981) vor allem dazu dienen, beiden Partnern ein angemessenes Problembewusstsein (bezüglich der Anteile beider Partner an der Aufrechterhaltung des Problems) zu vermitteln, aufzuzeigen, mit welchen Methoden dysfunktionales (kognitives, emotionales und behaviorales) Verhalten verändert werden kann und die Veränderungsmotivation dazu zu schaffen. Mittels Übungen, welche durch Prompting des Therapeuten einen maximalen Lerngewinn in kurzer Zeit ermöglichen, werden dem Paar neue Verhaltensweisen gelernt, welche dann im Alltag durch regelmässige 
Übungen (Hausaufgaben) verankert und gefestigt werden sollen. Auch wenn sich die Paartherapie zunehmend stärker mit der Rolle von Hausaufgaben zu befassen beginnt, besteht noch immer Forschungsbedarf, obgleich wenn davon ausgegangen werden kann, dass viele Erkenntnisse, welche im Individualbereich gewonnen wurden, auch auf Paartherapien extrapoliert werden können. Gerade jedoch die Dynamik von Paartherapien mit ihren multiplen Interaktionen zwischen den Partnern, jedem einzelnen Partner mit dem Therapeuten und dem Gesamtsystem lässt jedoch erwarten, dass die Sachlage häufig komplexer ist und daher auch Hausaufgaben in der Paartherapie einen komplizierteren Vorgang als in der Individualtherapie darstellen. Beide Partner sind jeweils zu motivieren, die Hausaufgaben symmetrisch auszuführen und der Therapeut ist angehalten, dieses Gleichgewicht behutsam zu wahren. Es ist daher wünschenswert, dass auch in der Paartherapie vermehrt Studien zu Hausaufgaben und deren Wirksamkeit durchgeführt werden. 


\section{Literatur}

Aponte, H. J. \& DiCesare, E. J. (2000). Structured theory. In F. M. Dattilio \& L. J. Bevilacqua (Eds.), Comparative treatments for relationship dysfunction (pp. 45-57). New York: Springer.

Baucom, D. H., Epstein, N. B., LaTaillade, J. J. \& Kirby, J. S. (2008). Cognitive-behavioral couple therapy. In A. S. Gurman (Eds.), Clinical hanbook of couple therapy $\left(4^{\text {th }}\right.$ Ed., pp. 31-72). New York: Guilford.

Baucom, Donald H., Shoham, V., Mueser, K. T., Daiuto, A. D. \& Stickle, T. R. (1998). Empirically supported couple and family interventions for marital distress and adult mental health problems. Journal of Consulting and Clinical Psychology, 66, 53-88.

Bauriedl, T., Cierpka, M., Neraal, T. \& Reich, G. (2002). Psychoanalytische Paar- und Familientherapie. In M. Wirsching \& P. Scheib (Hrsg.), Paar- und Familientherapie (S. 79-106). Berlin: Springer.

Bevilacqua, L. J. \& Dattilio, F. M. (2001). Brieffamily therapy homework planner. New York: Wiley.

Bodenmann, G. (2004). Verhaltenstherapie mit Paaren: Ein modernes Handbuch für die psychologische Beratung und Behandlung. Bern: Huber.

Bodenmann, G. (2005). Beziehungskrisen: Erkennen, verstehen und bewältigen (2. Aufl.). Bern: Huber.

Bodenmann, G. (2006). Stress und Partnerschaft: Gemeinsam den Alltag bewältigen (4. Aufl.). Bern: Huber.

Bodenmann, G. \& Fux Brändli, C. (2011). Was Paare stark macht (3. Aufl.). Zürich: Springer.

Bodenmann, G. \& Gabriel, B. (2008). Die therapeutische Beziehung in der Paartherapie. In M. Hermer \& B. Röhrle (Hrsg.), Handbuch der therapeutischen Beziehung (S. 13891412). Tübingen: DGTV. 
Bodenmann, G., Schär, M. \& Gmelch, S. (2008). Glücklich zu zweit trotz Alltagsstress. (DVD mit Manual). Zürich: Universität Zürich.

Bodenmann, G. \& Shantinath, S. D. (2004). The Couples Coping Enhancement Training (CCET): A new approach to prevention of marital distress based upon stress and coping. Family Relations, 53 (5), 477-484.

Bryant, M. J., Simons, A. D. \& Thase, M. E. (1999). Therapist skill and patient variables in homework compliance: Controlling an uncontrolled variable in cognitive therapy outcome research. Cognitive Therapy and Research, 23, 381-399.

Burns, D. D. \& Auerbach, A. H. (1992). Does homework compliance enhance recovery from depression. Psychiatric Annals, 22(9), 464-469.

Christensen, A. \& Heavey, C. L. (1999). Interventions for couples. Annual Review of Psychology, 50, 165-190.

Christensen, A., Atkins, D. C., Baucom, B. \& Yi, J. (2010). Marital status and satisfaction five years following a randomized clinical trial comparing traditional versus integrative behavioral couple therapy. Journal of Consulting and Clinical Psychology, 78, 225-235. Dattilio, F. M. (1998). Case studies in couple and family therapie: Systemic and cognitive perspectives. New York: Guilford.

Dattilio, F. M. (2002). Homework assignments in couple and family therapy. Journal of Clinical Psychology, 58, 535-547.

Dattilio, F. M. \& Beck, A. T. (2009). Cognitive-behavioral therapy with couples and families: a comprehensive guide for clinicians. New York: Guilford.

Dattilio, F. M. \& Dickson, J. (2007). Assigning homework to couples and families. Cognitive and Behavioral Practice, 14, 268-277.

Dattilio, F. M., Kazantzis, N., Shinkfield, G. \& Carr, A. G. (2011). A survey of homework use, experience of barriers to homework, and attitudes about the barriers to homework 
among couples and family therapists. Journal of Marital and Family Therapy, 37, 121-136.

De Jong, P. \& Berg, I. K. (2008). Interviewing for Solutions ( $3^{\text {th }}$ Ed.). Belmont, CA: Brooks Cole.

Dunn, R. L. \& Schwebel, A. I. (1995). Meta-analytic review of marital therapy outcome research. Journal of Family Psychology, 9, 58-68.

Epstein, N. B. (2010). Couples. In N. Kazantzis \& L. L'Abate (Eds.), Handbook of homework assignments in psychotherapy: Research, practice, and prevention (pp. 187-200). New York: Springer.

Epstein, N. B. \& Baucom, D. H. (2002). Enhance cognitive-behavioral therapy for couples: A contextual approach. Washington, D.C.: American Psychological Association.

Fehm, L. \& Fehm-Wolfsdorf, G. (2009). Therapeutische Hausaufgaben. In J. Margraf \& S. Schneider (Hrsg.), Lehrbuch der Verhaltenstherapie (3. Aufl., Bd. 1, S. 710-719). Heidelberg: Springer.

Gottman, J. M. (1994). What predicts divorce? Hillsdale, NJ: Erlbaum.

Greenberg, L. S. \& Goldman, R. N. (2010). Die Dynamik von Liebe und Macht. Emotionsfokussierte Paartherapie. München: Reinhardt.

Greenberg, L. S. \& Warwar, S. H. (2006). Homework in an emotion-focused approach to experiential therapy. Journal of Psychotherapy Integration, 16, 178-200.

Hargens, J. (2010). So kann's gelingen: Rahmen hilfreicher Gespräche im beraterischtherapeutischen Kontext. Dortmund: Borgmann.

Heekerens, H.-P. (2002). Die Funktionale Familientherapie. In S. K. D. Sulz \& H.-P. Heekerens (Hrsg.), Familien in Therapie. Grundlagen und Anwendung kognitivbehavioraler Familientherapie (S. 159-183). München: CIP-Medien.

Helbig, S. \& Fehm, L. (2005). Der Einsatz von Hausaufgaben in der Psychotherapie. Empfehlungen und ihre empirische Fundierung. Psychotherapeut, 50, 122-128. 
Hull, C. L. (1952). A behavior system: An introduction to behavior theory concerning the individual organism. New Haven: Yale University Press.

Imel, Z. E. \& Wampold, B. E. (2008). The importance of treatment and the science of common factors in psychotherapy. In S. D. Brown \& R. W. Lent (Eds.), Handbook of counseling psychology (4 ${ }^{\text {th }}$ Ed., pp. 249-256). New York: Wiley.

Jacobson, N. S., \& Christensen, A. (1996). Acceptance and change in couple therapy: A therapist's guide to transforming relationships. New York: Norton.

Johnson, S. M. (2004). The practice of emotionally focused couple therapy: Creating connection ( $2^{\text {nd }}$ Ed.). New York: Routledge.

Kazantzis, N. (2000). Power to detect homework effects in psychotherapy outcome research. Journal of Consulting and Clinical Psychology, 68, 166-170.

Kazantzis, N. \& L'Abate, L. (2006). Handbook of homework assignments in psychotherapy: Research, practice, and prevention. New York: Springer.

Kazantzis, N., Lampropoulos, G. K. \& Deane, F. P. (2005). A national survey of practicing psychologists' use and attitudes toward homework in psychotherapy. Journal of Consulting and Clinical Psychology, 73, 742-748.

Kazantzis, N., Whittington, C. \& Dattilio, F. M. (2010). Meta-analysis of homework effects in cognitive and behavioral therapy: A replication and extension. Clinical Psychology: Science and Practice, 17, 144-156.

L'Abate, L. (2007). Hugging, holding, huddling and cuddling (3HC): A task prescription in couple and family therapy. In L. L. Hecker \& C. Ford Sori (Eds.), The therapist's notbook (Vol. 2). New York: Haworth Press.

Luborsky, L., Rosenthal, R., Diguer, L., Andrusyna, T. P., Berman, J. S., Levitt ... Krause, E. D. (2002). The Dodo bird verdict is alive and well - Mostly. Clinical Psychology: Science and Practice, 9, 2-12. 
Maccoby, N. \& Solomon, D. S. (1981). Heart disease prevention: Community studies. In R. E. Rice \& W. J. Paisley (Eds.), Public communication campaigns (pp. 105-126). Beverly Hills, CA: Sage.

Messer, S. B. \& Wampold, B. E. (2002). Let's face facts: Common factors are more potent than specific therapy ingredients. Clinical Psychology: Science and Practice, 9, 21-25.

Schindler, L., Hahlweg, K. \& Revenstorf, D. (1998). Partnerschaftsprobleme: Diagnose und Therapie (2. Aufl.). Berlin: Springer.

Schindler, L., Hahlweg, K. \& Revenstorf, D. (1999). Partnerschaftsprobleme. Möglichkeiten zur Bewältigung. Ein Handbuch für Paare (2. Aufl.). Berlin: Springer.

Shadish, W. R. \& Baldwin, S. A. (2003). Meta-analysis of MFT interventions. Journal of Marital and Family Therapy, 29, 547-570.

Shadish, W. R. \& Baldwin, S. A. (2005). Effects of behavioral marital therapy: A metaanalysis of randomized controlled trials. Journal of Consulting and Clinical Psychology, 73, 6-14.

Shadish, W. R., Montgomery, L. M., Wilson, P., Wilson, M. R., Bright, I. \& Okwumabua, T. (1993). Effects of family and marital psychotherapies: A meta-analysis. Journal of Consulting and Clinical Psychology, 61, 992-1002.

Sprenkle, D. H., Davis, S. D. \& Lebow, J. (2009). Common factors in couple and family therapy: The overlooked foundation for effective practice. New York: Guilford.

Thurmaier, F., Engl, J., Eckert, V. \& Hahlweg, K. (1992). Prävention von Ehe- und Partnerschaftsstörungen EPL (Ehevorbereitung - Ein Partnerschaftliches Lernprogramm). Verhaltenstherapie, 2, 116-124.

Tompkins, M. A. (2002). Guidelines for enhancing homework compliance. Journal of Clinical Psychology, 58, 565-576.

Weber, W. (1994). Wege zum helfenden Gespräch. Gesprächspsychotherapie in der Praxis (10. Aufl.). München: Reinhardt. 
Wuschel, I., \& Linden, M. (2008). Hausaufgaben. In M. Linden \& M. Hautzinger (Hrsg.), Verhaltenstherapiemanual (6. Aufl., S. 178-181). Heidelberg: Springer.

Prof. Dr. Guy Bodenmann

Universität Zürich

Psychologisches Institut

Binzmühlestrasse 14/Box 23

CH 8050 Zürich

Email: guy.bodenmann@psychologie.uzh.ch 